\title{
Relationship between Microtubule Network Structure and Intracellular Transport in Cultured Endothelial Cells Affected by Shear Stress*
}

\author{
Susumu KUDO $^{* *}$, Kenji IKEZAWA***, Mariko IKEDA*** and Kazuo TANISHITA ${ }^{\dagger}$
}

\begin{abstract}
Endothelial cells (ECs) that line the inner surface of blood vessels are barriers to the transport of various substances into or from vessel walls, and are continuously exposed to shear stress induced by blood flow in vivo. Shear stress affects the cytoskeleton (e.g., microtubules, microfilaments, intermediate filaments), and affects the transport of macromolecules. Here, the relationship between the microtubule network structure and this transport process for albumin uptake within cultured aortic endothelial cells affected by shear stress was studied. Based on fluorescent images of albumin uptake obtained by using confocal laser scanning microscopy (CLSM), both the microtubule network and albumin uptake in ECs were disrupted by colchicine and were affected by shear stress loading.
\end{abstract}

Key Words: Biological Engineering, Shear Flow, Bio-Fluid Mechanics, Endothelial Cells, Transport, Albumin, Microtubule, Vesicle

\section{Introduction}

Atherosclerotic lesions occur preferentially at low shear-stress regions located at the wall of bifurcations and at the inner curved-surface of arteries ${ }^{(1)}$. In these regions, macromolecules, such as low-density lipoprotein, albumin, and horse-radish peroxidase (HRP), accumulate in the intima of arterial walls ${ }^{(2)-(4)}$.

Endothelial cells (ECs) that line the inner surface of blood vessels are barriers to the transport of various substances into or from vessel walls, and thus regulate the exchange of molecules between blood and vessel walls. ECs are continuously exposed to shear stress by blood in vivo. Shear stress affects the physiological and biochemical processes in ECs ${ }^{(5),(6)}$. In particular, transport across an EC is

* Received 26th May, 2004 (No. 04-4130)

** Department of Mechanical Engineering, Shibaura Institute of Technology, 3-9-14 Shibaura, Minato-ku, Tokyo 1088548, Japan. E-mail: kudous@ sic.shibaura-it.ac.jp

*** Institute of Biomedical Engineering, Keio University, 314-1 Hiyoshi, Kohoku-ku, Yokohama 223-8522, Japan

**** Keio Leading-edge Laboratory of Science and Technology, Keio University, 3-14-1 Hiyoshi, Kohoku-ku, Yokohama 223-8522, Japan

$\dagger$ Department of System Design Engineering, Keio University, 3-14-1 Hiyoshi, Kohoku-ku, Yokohama 223-8522, Japan affected by steady shear stress. For example, steady shear stress increases the uptake of low-density lipoprotein into $\mathrm{ECs}^{(7)}$, and step changes in shear stress increase the uptake of $\operatorname{HRP}^{(8)}$. Shear stress also affects the transendothelial permeability to macromolecules. For example, the permeability of albumin across an endothelial monolayer is increased by steady shear stress ${ }^{(9)}$, and that of albumin and dextran is increased after $48 \mathrm{hr}$ of shear stress loading ${ }^{(10)}$. In a previous study, we found that albumin uptake into cultured ECs is increased by $30 \%$ when the ECs are exposed to low shear stress (between 0.5 and $1 \mathrm{~Pa}$ ), but gradually decreases when exposed to high shear stress (between 2 to $8 \mathrm{~Pa})^{(11)}$. Despite such studies, the mechanism of the shear-dependent transport process is not yet thoroughly understood.

Pathways for macromolecule transport across endothelium can be classified roughly into two types: intercellular transport and intracellular transport ${ }^{(12),(13)}$. Numerous studies have showed that both transport pathways coexist ${ }^{(14)-(16)}$, indicating the importnce of both pathways in macromolecule transport. Paracellular and transcellular pathways contribute to the transport of substances across ECs ${ }^{(12)-(15)}$. Paracellular transport is a passive transport involving diffusion and convection through the junctions between cells. In contrast, transcellular transport is an energy-dependent transport by transcytosis. Transendothelial permeability to macromolecules 
such as albumin remains under debate, however ${ }^{(17),(18)}$. For example, Schnitzer and $\mathrm{Oh}^{(15)}$ investigated the inhibition of different aspects of bovine serum albumin transport across bovine lung EC monolayer, and suggested that albumin transport could be $30-40 \%$ paracellular transport, $10-20 \%$ fluid-phase transcellular transport, and 50\% albondin-mediated transcellular transport (albondin: $60 \mathrm{kDa}$ albumin-binding protein). Therefore, in this study, we focused on the intracellular transport pathway in ECs.

Many studies on ECs indicate that vesicular transport requires the organization of cytoskeleton, such as microtubules and microfilaments ${ }^{(19),(20)}$. Microtubules are depolymerized by microtubule-disruption agents, such as colchicine and nocodazole, and by cold temperature $\left(-2^{\circ} \mathrm{C}\right)^{(21)}$, and thus alter the microtubule network structure. In aortic ECs, effects of colchicine on intracellular macromolecular transport include the reduction of both the endocytic activity ${ }^{(22)}$ and transcytosis ${ }^{(23)}$. If the change of vesicular transport process is caused by change of the microtubule network structure induced by shear stress, then understanding the relationship between the microtubule network structure and vesicular transport of macromolecules inside an EC is critical in clarifying the mechanisms of such aberrations.

We therefore studied the effect of the microtubule network structure on the vesicular transport process for albumin uptake within cultured porcine aortic ECs (PAECs). In this in vitro study, first we investigated the effect of colchicine-induced disruption of the microtubule network structure on the vesicular transport process for albumin uptake. This effect was studied by fluorescent images of albumin uptake and microtubules at various colchicine concentrations. Then we investigated if the vesicular transport process for albumin uptake induced by shear stress is related to the microtubule network structure.

\section{Materials and Methods}

\subsection{Culture media, preparation of fluorescent la- bels, and immunofluorescence}

Dulbecco's Modified Eagle Medium (D-MEM), Trypsin-EDTA, and Antibiotic-Antimycotic (composed of penicillin, streptomycin, and amphotericin B) were obtained from GIBCO (Grand Island, NY). TRITC-albumin and a secondary antibody, tetramethylrhodamine goat anti-mouse $\operatorname{IgG}(\mathrm{H}+\mathrm{L})$, were purchased from Molecular Probes, Inc. (Eugene, OR). Bovine serum albumin (BSA) was obtained from Sigma Chemical Co. (St. Louis, MO). Fetal bovine serum (FBS) was obtained from JRH Bioscience (Lenexa, KS). Dulbecco's PBS (-) was from Nissui Pharmaceutical Co., Ltd. (Tokyo). A primary antibody, Mouse Anti- $\beta$-Tubulin Monoclonal (Clone; DM1B), was purchased from ICN Biochemicals, Inc. (Aurora, Ohio). $\mathrm{MgCl}_{2} \cdot 6 \mathrm{H}_{2} \mathrm{O}$ and $\mathrm{CaCl}_{2}$ were obtained from Wako Pure Chemical Industries, Ltd. (Osaka). Paraformalde- hyde, colchicine, and Triton X-100 were obtained from Junsei Chemical Co., Ltd. (Tokyo).

\subsection{Culture method}

Porcine aortic endothelial cells (PAECs) were collected by a modified method developed by Shasby and Shasby ${ }^{(17)}$. In brief, a porcine aorta was obtained from a slaughterhouse and preserved in ice-cold phosphatebuffered saline (PBS (-)) containing $200 \mathrm{U} / \mathrm{mL}$ penicillin, $200 \mu \mathrm{g} / \mathrm{mL}$ streptomycin, and $0.5 \mu \mathrm{g} / \mathrm{mL}$ amphotericin B. The aorta was opened longitudinally and the intima was rinsed with PBS (-). The lumen was gently scraped with a scalpel blade to isolate PAECs. The isolated PAECs were then suspended in culture medium (composed of D-MEM, $100 \mathrm{U} / \mathrm{mL}$ penicillin, $100 \mu \mathrm{g} / \mathrm{mL}$ streptomycin, $0.25 \mu \mathrm{g} / \mathrm{mL}$ amphotericin B, and 10\% FBS). They were then seeded in $25 \mathrm{~cm}^{2}$ tissue culture flasks (FALCON, Franklin Lakes, NJ) in culture medium, and cultured at $37^{\circ} \mathrm{C}$ in an incubator with $5 \% \mathrm{CO}_{2}$ and $95 \%$ air. After confirming that PAECs had adhered to the bottom of the flasks, we replaced the culture medium with fresh medium. The PAECs were grown as primary culture. After 5-7 days, the cells were passaged at a 4-to- 1 split ratio by treatment with $0.05 \%$ trypsin and $0.53 \mathrm{mM}$ EDTA.4Na. For the albumin-uptake experiments and the microtubule staining, PAECs were grown to confluence on cover slips and used from 5th to 9 th passage.

\subsection{Flow circuit}

The PAECs were cultured on clean coverslips $(22 \mathrm{~mm}$ in diameter). After the PAECs were confluent, the cells on the coverslips were placed in a parallel-plate flow chamber $(0.2 \mathrm{~mm}$ high, $20 \mathrm{~mm}$ wide, and $90 \mathrm{~mm}$ long) for shearstress loading (Fig. 1). The flow circuit was filled with DMEM containing $10 \%$ FBS, and the steady laminar flow was generated by a peristaltic pump. A depulsator was used to eliminate the pulsatile of the flow. To maintain a medium $\mathrm{pH}$ of 7.4 , a dilution gas of $5 \% \mathrm{CO}_{2}-95 \%$ air was introduced into a reservoir and then mixed with the DMEM flow as needed. Temperature in the system, except the peristaltic pump, was maintained at $37^{\circ} \mathrm{C}$ by using a water bath. With this flow circuit, the PAECs were subjected to either a low shear stress $(1 \mathrm{~Pa})$ or a high shear stress $(6 \mathrm{~Pa})$ for $48 \mathrm{hr}$. The low and high shear stress was calculated as follows; $\tau=6 \mu Q / b h^{2}$, where $\mu$ $(0.00085 \mathrm{Pas})$ is the viscosity of the DMEM containing FBS $10 \%$ at $37^{\circ} \mathrm{C} ; Q$ is volume flow $\left(0.16\right.$ and $9.4 \mathrm{~cm}^{3} / \mathrm{s}$ at 1 and $6 \mathrm{~Pa}$, respectively); $b(2 \mathrm{~cm})$ is the cross-sectional width of the flow path; $a(0.02 \mathrm{~cm})$ is the distance between the cells on the upper side of coverslips and the upper plate of flow chamber.; Reynolds number is 9.2 and 55 at 1 and $6 \mathrm{~Pa}$, respectively.

\subsection{TRITC-albumin uptake}

After shear stress loading for $48 \mathrm{hr}$, the coverslips with the PAECs were removed from the flow chamber, then soaked in PBS including TRITC-albumin 


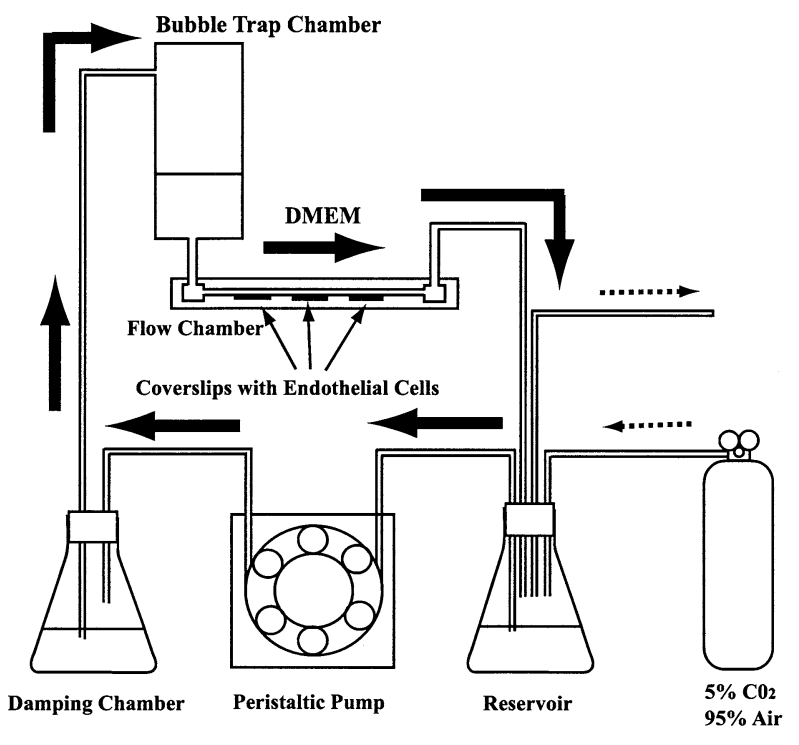

Fig. 1 Schematic of flow circuit for producing shear stress on endothelial cells. Endothelial cells on upper side of coverslips were placed in the flow chamber, and then subjected to shear stress (i.e., flow) for $48 \mathrm{hr}$. The calculation of shear stress $(\tau)$ is mentioned under Materials and Methods.

$(0.1 \mathrm{mg} / \mathrm{mL}$ of tetramethylrhodamine conjugated albumin, Molecular Probes) and albumin $(0.3 \mathrm{mg} / \mathrm{mL}$, Sigma) prewarmed at $37^{\circ} \mathrm{C}$, and then incubated for $120 \mathrm{~min}$. The PAECs were rinsed three times with $2 \mathrm{~mL}$ of PBS, fixed in $2 \%$ parafolmaldehyde for $20 \mathrm{~min}$, and then again rinsed three times with $2 \mathrm{~mL}$ of PBS. Fluorescent and phase contrast images of the PAECs were obtained by using a CLSM (MRC-600, Bio-Rad Microscience) mounted on an inverted microscope (TMD 300, Nikon) with a NCF Plan Apo DM 63 X objective lens (NA 1.4). The excitation wavelength was $514 \mathrm{~nm}$ from a $25-\mathrm{mW}$ argon laser. The TRITC-albumin was visualized using a 509- to 519$\mathrm{nm}$ band-pass excitation filter, a 540-nm diachronic mirror, and a 550-nm long-pass emission filter.

\subsection{Effects of colchicine on albumin uptake}

Colchicine was dissolved in culture medium and albumin-uptake solution at various concentrations (10$100 \mu \mathrm{M})$. Before each albumin-uptake experiment, the PAEC monolayer was treated with colchicine in culture medium at $37^{\circ} \mathrm{C}$ for $60 \mathrm{~min}$.

\subsection{Microtubule staining}

The PAEC monolayer was washed in $37^{\circ} \mathrm{C}$ PBS (+) three times, and then fixed in $2 \%$ paraformaldehyde for $20 \mathrm{~min}$ at room temperature. For cell permeabilization, this fixed monolayer was incubated in $0.5 \%$ Triton X-100 in PBS (+) for $5 \mathrm{~min}$ at room temperature.

For immunofluorescence of the microtubules, the primary antibody (Mouse Anti- $\beta$-Tubulin Monoclonal) and secondary antibody (tetramethylrhodamine goat antimouse $\operatorname{IgG}(\mathrm{H}+\mathrm{L}))$ were diluted to $1: 10$ and $1: 100$, re- spectively, in PBS (-) containing 1\% BSA. Permeabilized cells were incubated with the primary antibody for $40 \mathrm{~min}$ at $37^{\circ} \mathrm{C}$, washed in PBS (+) three times at room temperature, and then incubated with the secondary antibody for $40 \mathrm{~min}$ at $37^{\circ} \mathrm{C}$. Negative controls were similarly prepared, except the primary antibody was replaced by PBS $(+)$ containing $38.5 \mathrm{mg} / \mathrm{mL}$ BSA.

\subsection{Image analysis}

Albumin-uptake images were analyzed by using a UMAX Pulsar 2250 computer and NIH Image Program (public domain software). A projection image for this analysis was constructed from the images taken from a series of focal planes. For the image construction, we used the brightest-point projection method, where the brightest point encountered in the same position of each focal plane was projected onto an image.

For determining the albumin uptake, we detected fluorescent particles in a projection image by the following procedure. First, we used the Sobel edge-detection (in the NIH Image software) to detect edges of fluorescent particles in the original gray-scale image (a projection image; 8-bits) obtained by CLSM. Then, that image that contained detected particles was then segmented into particles and other components, which mainly consisted of background, by using a threshold technique. In this technique, the threshold was automatically set based on analysis of the histogram of the entire image. We defined an Image A as the threshold image of detected particles. In Image A, all of the pixels of particles above the threshold were set to white and all other pixels to black. The values of black and white pixels were 255 and 0 , respectively. In Image A, the pixel values of particles were ignored, and thus the localization and size of the particles were available for image analysis. From the original gray-scale image, we then quantified the fluorescent intensity of the same particles as in Image A.

\subsection{Statistical analysis}

Statistical comparisons were done by using one-way analysis of variance (ANOVA), and differences among two groups were calculated using Scheffé's post hoc tests. A difference at $P<0.05$ level was judged as significant. The data are presented in terms of mean $\pm \mathrm{SE}$.

\section{Results}

\subsection{Effect of colchicine on microtubule network in ECs}

Our experiments showed that colchicine disrupted the microtubule network structure, and that the extent of disruption depended on the colchicine concentration. In control PAECs (no treatment with colchicine), microtubules were organized radially (Fig. 2(a)). In PAECs treated with $20 \mu \mathrm{M}$ colchicine, such radial structure was disrupted (Fig. 2 (b)). In PAECs treated with $100 \mu \mathrm{M}$ colchicine, microtubules were completely depolymerized and PAECs 


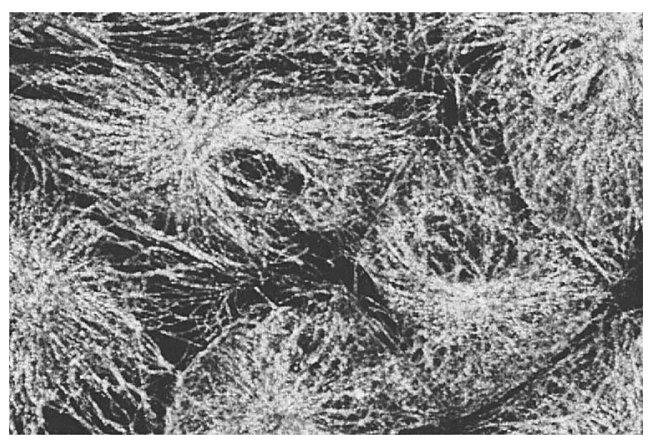

(a) Control

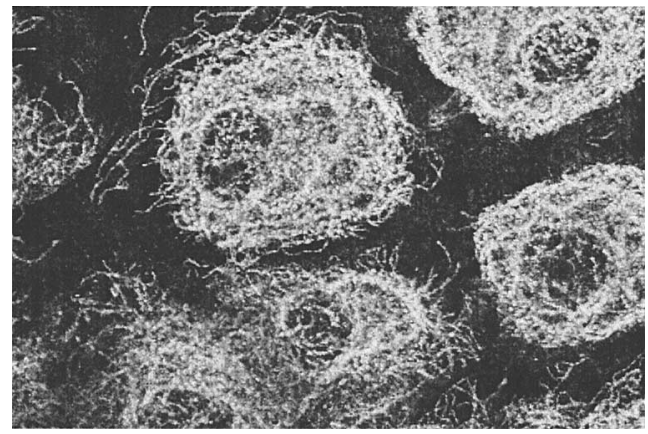

(b) $20 \mu \mathrm{M}$

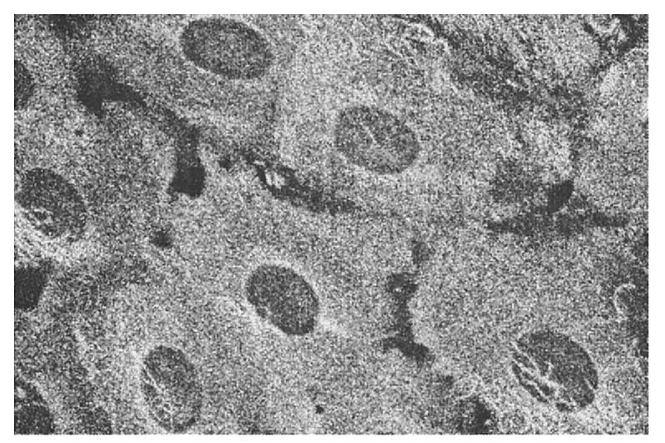

$20 \mu \mathrm{m}$

(c) $100 \mu \mathrm{M}$

Fig. 2 Fluorescent images showing effect of colchicine on microtubule structure in PAECs. (a): control $(0 \mu \mathrm{M}$ colchicine), (b): $20 \mu \mathrm{M},(\mathrm{c}): 100 \mu \mathrm{M}$

showed fine fibrillar tubulin patterns (Fig. 2(c)).

\subsection{Effect of colchicine on albumin uptake into} PAECs

To confirm if the microtubule network affected the albumin uptake into PAECs, we investigated the effect of colchicine on albumin uptake into PAECs by obtaining fluorescence images of TRITC-albumin taken up into PAECs. The fluorescence image in Fig. 3 reveals that TRITC-albumin (bright fluorescent spots) accumulated in the cellular compartment in PAECs, which is consistent with our previous study ${ }^{(11)}$, and that albumin taken up into PAECs decreased with increasing colchicine concentration. From these images, we quantified the albumin uptake into PAECs incubated in different concentrations of colchicine for different incubation times. In Fig. 4,

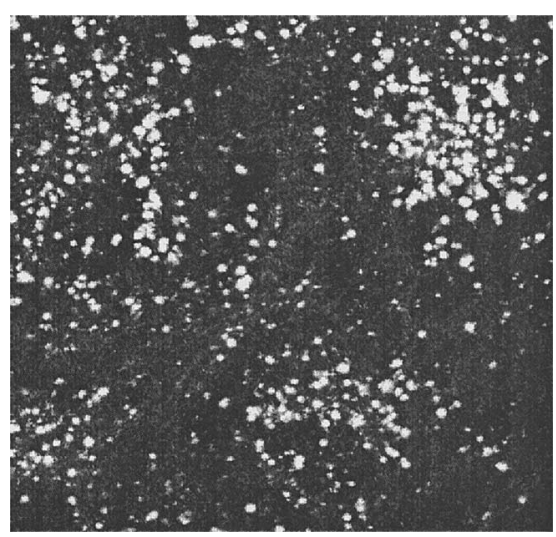

(a) Control

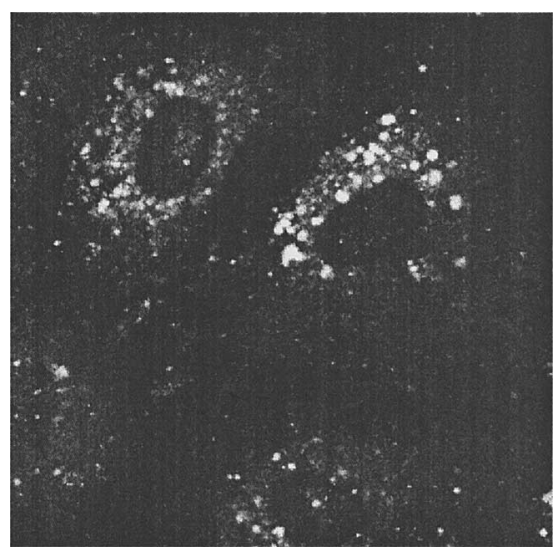

(b) $20 \mu \mathrm{M}$ colchicine

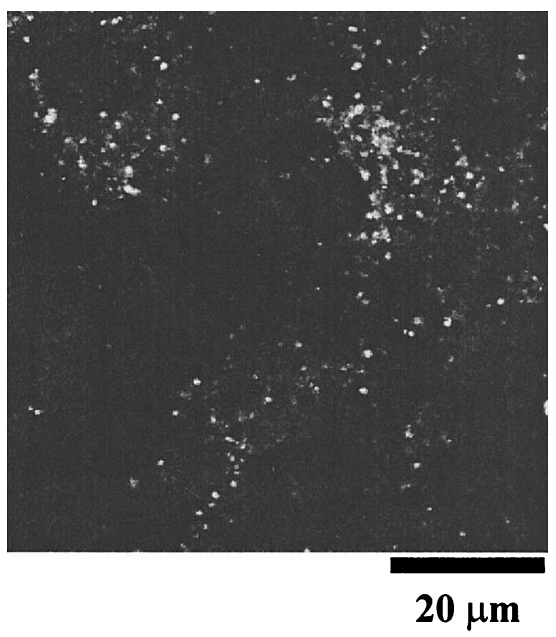

(c) $100 \mu \mathrm{M}$ colchicine

Fig. 3 Fluorescent images showing effect of colchicine on TRITC-albumin uptake into PAECs. (a): control (no-treatment with colchicine), (b): $20 \mu \mathrm{M}$ colchicine, (c): $100 \mu \mathrm{M}$ colchicine

the relative albumin uptake was normalized by the uptake for the control PAECs (no-treatment with colchicine) at $120 \mathrm{~min}$. This figure shows that the albumin uptake increased with increasing incubation time and that treatment with colchicine inhibited this uptake. For example, at 120min incubation time in $10 \mu \mathrm{M}, 20 \mu \mathrm{M}, 40 \mu \mathrm{M}$, and $100 \mu \mathrm{M}$ 


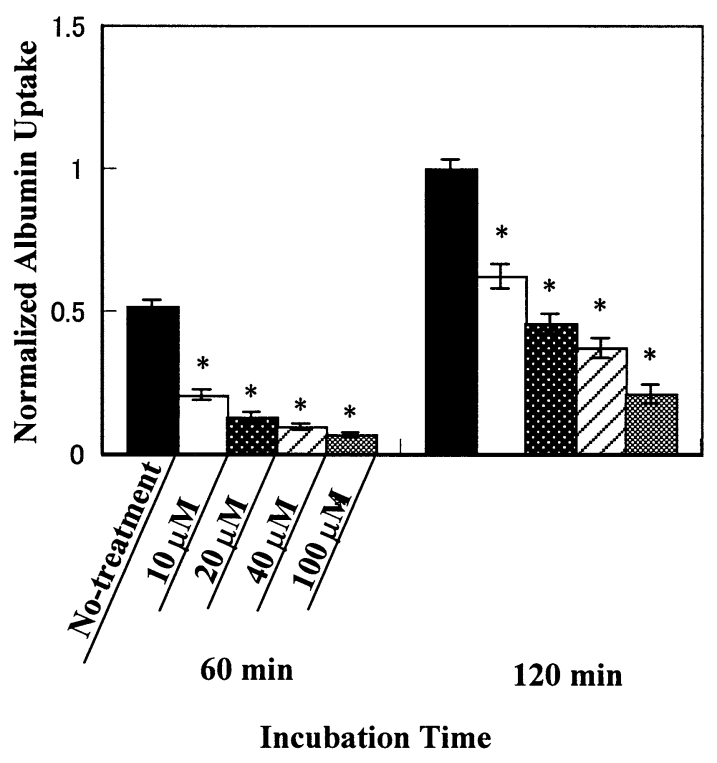

Fig. 4 Effect of colchicine concentration and incubation time on TRITC-albumin uptake into PAECs. Values are means \pm SE. The number of experiments is 10 in each condition. ${ }^{*} p<0.05$ compared with $0 \mu \mathrm{M}$ at same incubation time.

colchicine, albumin uptake decreased to $62 \%, 46 \%, 37 \%$, and $21 \%$, respectively. To further investigate the relationship between the microtubule network and albumin uptake, we obtained fluorescence images of both the network and albumin in the same cells. From this fluorescent image (Fig. 5), the intracellular compartment containing TRITCalbumin (red spots) was localized on the microtubule network structure (green structure).

\subsection{Effect of shear stress on microtubule network structure}

The effect of shear stress on the microtubule network structure is clearly evident in the fluorescent images in Fig. 6. Under no-shear conditions, microtubules were organized radially. Although the PAECs were elongated after shear stress loading, the cells did not significantly orient to the flow direction. Microtubules were oriented along the long axis of the cell after low (1 Pa) and high $(6 \mathrm{~Pa})$ shear stress loading. Both low and high levels of shear stress clearly changed the microtubule network structure. However, no significant differences in microtubule structure were evident between the low and high shear-stress conditions.

\subsection{Relationship between microtubule network structure and albumin uptake}

The effect of shear stress on albumin uptake into the PAECs is clearly evident in Fig. 7. In Fig. 7, the relative albumin uptake was normalized by the uptake for control PAECs under no-shear conditions. At low shear stress (1 Pa), the albumin uptake increased by $100 \%$ compared with that in the static PAECs, whereas at high shear stress (6 $\mathrm{Pa})$, the albumin uptake decreased by $50 \%$. After $48 \mathrm{hr}$

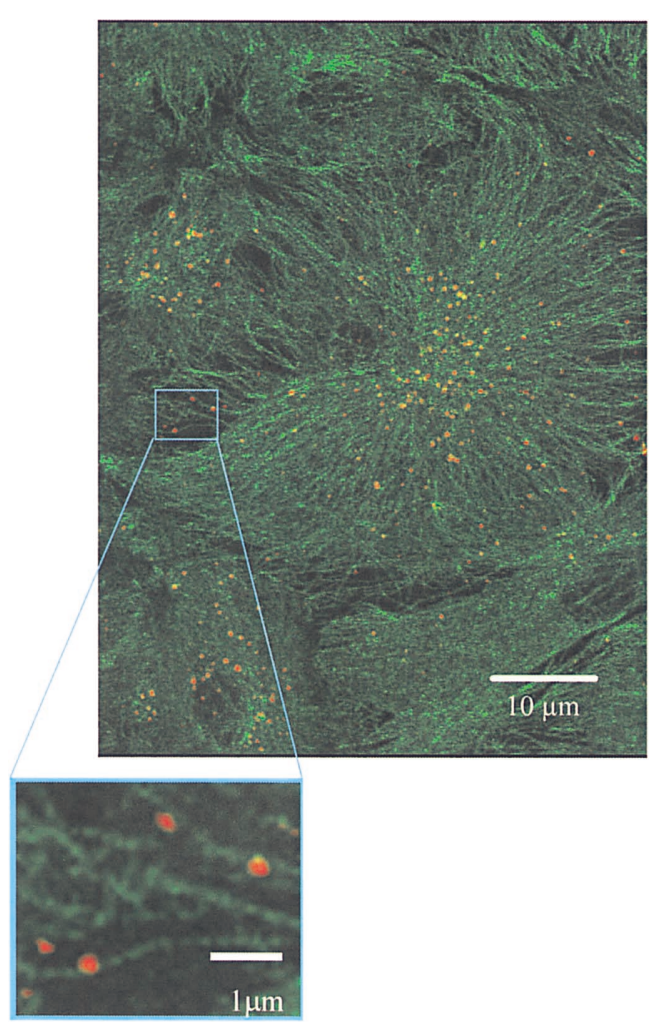

Fig. 5 Fluorescent images of both microtubule network and TRITC albumin in the same cells without addition of colchicine. TRITC albumin (red spots) is localized on the microtubule network structure (green structure).

of shear stress loading, the PAECs were incubated with colchicine for $60 \mathrm{~min}$, and then incubated with TRITCalbumin and colchicine. Colchicine depressed the albumin uptake under all three shear-stress conditions (no, low, or high shear stress). The effect was particularly pronounced in low shear stress conditions, where a colchicine concentration of $50 \mu \mathrm{M}$ decreased the albumin uptake by $90 \%$. At a colchicine concentration of $50 \mu \mathrm{M}$, the albumin uptake was similar for all three shear-stress conditions. These results suggest that the microtubule network structure is strongly related to the albumin uptake into PAECs affected by shear stress.

\section{Discussion}

Our results indicate that microtubules are involved in albumin uptake into PAECs and that microtubules are strongly related to the albumin uptake into PAECs affected by shear stress. Although the PAECs exhibited elongated cell shape, the cells did not significantly orient to the flow direction after $48 \mathrm{hr}$ shear stress loading. This result was not consistent with many other studies that showed cell alignment with flow direction. It is considered that endothelial cells begin to elongate prior to beginning to align with the flow direction ${ }^{(24)}$. In our study, the PAECs is considered to be under aligning with flow direction. The difference between our result and other studies might be 


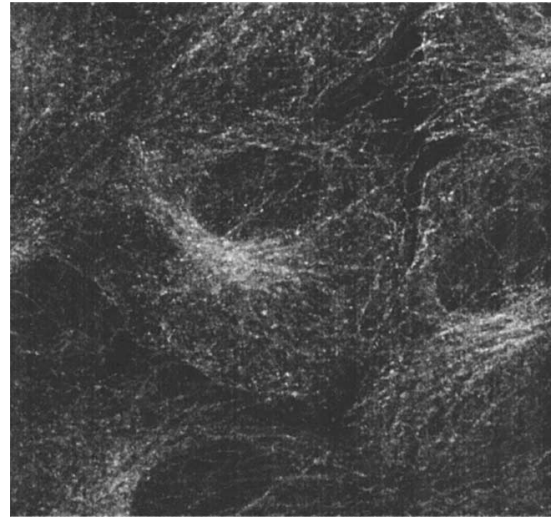

(a) No flow

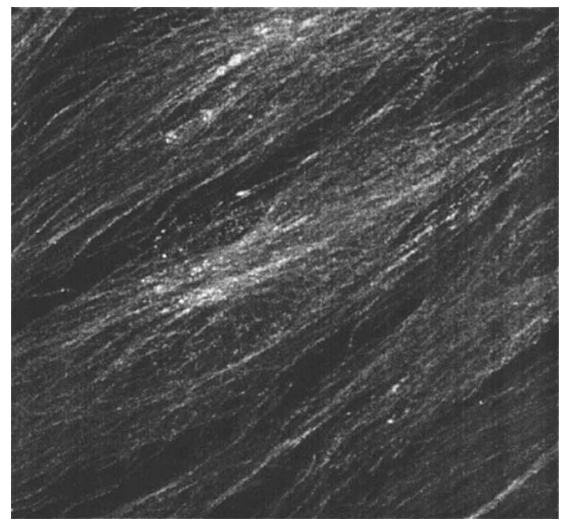

(b) $1 \mathrm{~Pa}$

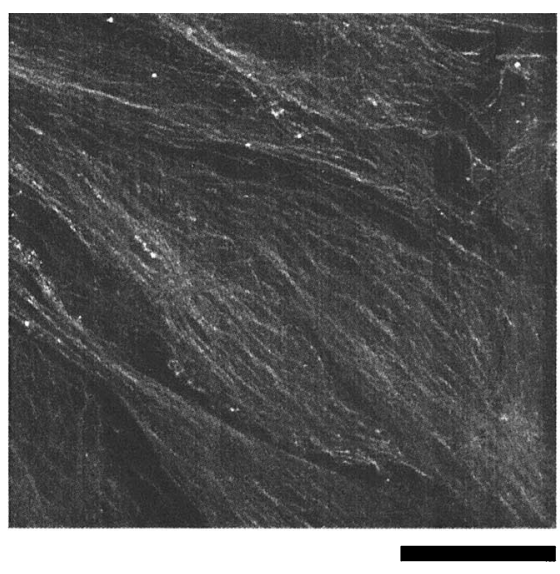

$20 \mu \mathbf{m}$

\section{Flow Direction}

(c) $6 \mathrm{~Pa}$

Fig. 6 Fluorescent images showing effect of shear stress on microtubule network structure in PAECs after shear stress loading for $48 \mathrm{hr}$. (a): No flow, (b): low shear stress $(1 \mathrm{~Pa}),(\mathrm{c})$ : high shear stress $(6 \mathrm{~Pa})$

due to different type of endothelial cells and different culture media including serum. We used porcine endothelial cells. On the other hand, bovine and human endothelial cells were used in most of other studies.

Vesicles mediate macromolecules uptake into ECs

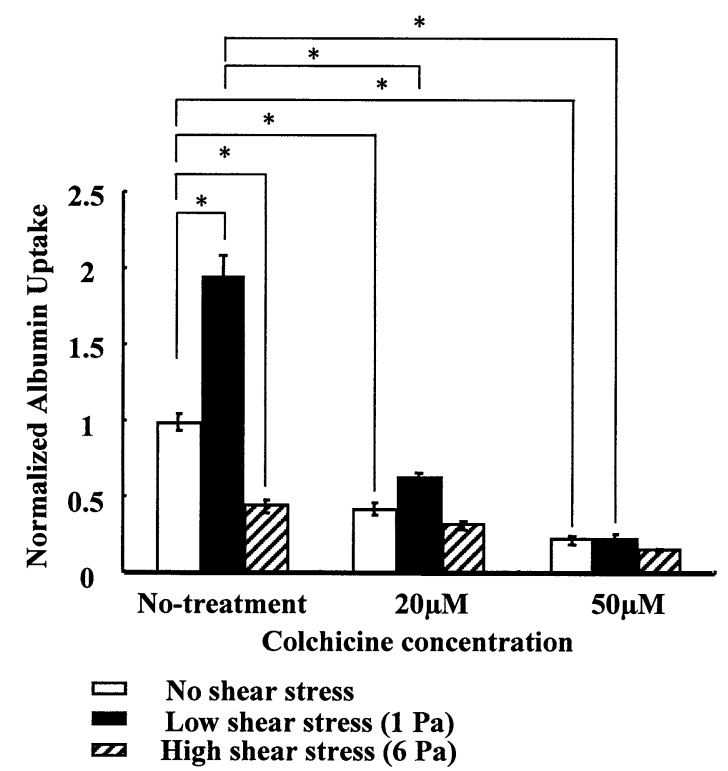

Fig. 7 Histogram showing effect of shear stress and colchicine on TRITC-albumin uptake into PAECs. Values are means \pm SE. The number of experiments is 7 in each condition. *significant $(p<0.05)$

and deliver the macromolecules to endosomes and lysosomes $^{(12)}$. Thus, macromolecules taken up into ECs exist in the cellular compartments such as vesicles, endosomes, and lysosomes. Due to fusion and dissociation of the compartments, it is difficult to discriminate the compartments from each other ${ }^{(25)}$. Discriminating them in fluorescent images (Fig. 3) is also difficult. Based on Figs. 3 and 4, vesicular transport for albumin uptake was inhibited by colchicine because colchicine reduced the accumulation of TRITC-albumin. In the transport process involved in the albumin uptake in PAECs, the motility of a vesicle is considered inhibited by disruption of the microtubule structure.

Schnitzer et al. ${ }^{(15)}$ showed that non-coated vesicles, known as caveolae vesicles, mediate the albumin uptake into ECs. Mundy et al. ${ }^{(26)}$ investigated the trafficking of caveolin-1-GFP (marker for caveolae) in stably expressing $\mathrm{CHO}$ cells. Mundy et al. found caveolin-1-GFP in vesiclelike structures that exhibited rapid movement along curvilinear paths within the cytoplasm and found that almost all caveolin-1-GFP movement ceased by treatment with nocodazole to depolymerize microtubules. These results by Mundy et al. suggest that caveolar vesicles are translocated along microtubules. In our study, the compartment containing TRITC-albumin was localized on microtubules (Fig. 5), and disruption of microtubules depressed the albumin uptake (Figs. 3 and 4). Our results suggest that the vesicles containing TRITC-albumin are transported along microtubules in PAECs.

We also investigated the relationship between microtubule network structure and albumin uptake into PAECs 
after shear stress loading. In a previous study ${ }^{(11)}$, we investigated albumin uptake into PAECs after $48 \mathrm{hr}$ of shear stress loading, and showed that at low shear stress (1 Pa), the albumin uptake increased by $30 \%$ and at high shear stress $(6 \mathrm{~Pa})$, the uptake decreased by $70 \%$. Results from our current study showed similar shear-dependent uptake; without addition of colchicine, the albumin uptake increased at low shear stress $(1 \mathrm{~Pa})$ and decreased at high shear stress $(6 \mathrm{~Pa})$. In both low and high shear-stress conditions, the flow-induced albumin uptake decreased with addition of colchicine. At a colchicine concentration of $50 \mu \mathrm{M}$, the albumin uptake was similar for all three shearstress conditions (no, low, and high shear stress), suggesting that microtubules are essential for albumin uptake into ECs affected by shear stress. However, no difference in microtubule structure was observed between low and high shear-stress conditions (Fig. 6).

Murray et al. ${ }^{(27)}$ investigated the ATP-dependent movement of endocytic vesicles in vitro, and showed that endosomes containing asialoorosomcoid bind to microtubules and are translocated along these microtubules in the presence of ATP by the system to observe both endocytic processing and microtubule movement. Bananis et al. ${ }^{(28)}$ investigated microtubule and motor-dependent endocytic vesicles in vitro, and showed that presegregation vesicles must bind to microtubules and move upon addition of ATP. They also showed colocalization of endocytic vesicles with kinesin, but not with cytoplasmic dynein, and showed that motility of vesicles is prevented by addition of $5^{\prime}$-adenylylimido-diphosphate (AMP-PNP, an inhibitor of kinesins). These results by Murray et al. and Bananis et al. suggest that kinesin on the microtubules and ATP play a key role in controlling the intracellular transport process.

In our previous study, we reported the effect of temperature and metabolic inhibitor on the albumin uptake into $\mathrm{ECs}^{(29)}$. At cold temperature $\left(4^{\circ} \mathrm{C}\right)$, the albumin uptake into an EC was inhibited completely, and after FCCP (metabolic inhibitor) treatment the uptake was also inhibited completely. These results show that intracellular transport depends on the energy (ATP) condition of the cell. Because mitochondrial membrane potential is considered to reflect the ability of ATP synthesis, we previously investigated the mitochondrial membrane potential and ATP synthesis after shear-stress loading ${ }^{(30)}$. The mitochondrial membrane potential was high at a low shear stress of $1 \mathrm{~Pa}$ and was low at a high shear stress of $6 \mathrm{~Pa}$, suggesting that the ATP synthesis of an EC decreases with increasing shear stress.

Results from our current study reveal the following mechanism for the intracellular transport pathway. Under low shear-stress conditions, TRITC-containing vesicles that bind to microtubules through kinesin move actively because ATP synthesis is high. As a result, albumin uptake is high under low shear-stress conditions. On the other hand, under high shear-stress conditions, the motility of TRITC-containing vesicles is reduced because ATP synthesis is low under such stress conditions. Thus, albumin uptake is low under high shear-stress conditions.

\section{Conclusions}

Our study indicated that albumin uptake into PAECs under no-flow conditions was inhibited by colchicine and the albumin taken up was localized on microtubles. Our study also showed that albumin uptake was shear stress dependent. These results suggest that albumin is translocated along microtubules in cultured PAEC monolayers under no-flow (no shear stress) conditions, and that this translocation is shear dependent and is related to the microtubules.

\section{Acknowledgment}

This study was supported in part by a Research Project of Academic Frontier from Japan's Ministry of Education, Culture, Sports, Science, and Technology, and by Grant-in-Aid for Scientific Research on Priority Areas from the Ministry of Education, Culture, Sports, Science and Technology, Japan, "Biomechanics at Micro- and Nanoscale Levels".

\section{References}

( 1 ) Caro, C.G., Fitz-Gerald, J.M. and Schroter, R.C., Arterial Wall Shear and Distribution of Early Atheroma in Man, Nature, Vol.223 (1969), pp.1159-1161.

( 2 ) Bafakat, A.I., Uhthoff, P.A.F. and Colton, C.K., Topographical Mapping of Sites of Enhanced HRP Permeability in the Normal Rabbit Aorta, J. Biomech. Eng., Vol.114 (1992), pp.283-292.

( 3 ) Packham, M.A., Rowsell, H.C., Jorgensen, L. and Mustard, J.F., Localized Protein Accumulation in the Wall of the Aorta, Exptl. Molec. Pathol., Vol.7 (1967), pp.214-232.

( 4 ) Schwanke, D.C. and Carew, T.C., Initiation of Atherosclerotic Lesions in Cholesterol-Fed Rabbits, Arteriosclerosis, Vol.9 (1989), pp.895-907.

( 5 ) Ando, J. and Kamiya, A., Blood Flow and Vascular Endothelial Cell Function, Front. Med. Biol. Eng., Vol.5 (1993), pp.245-264.

( 6 ) Nerem, R.M., Harrison, D.G., Taylor, W.R. and Alexander, R.W., Hemodynamics and Vascular Endothelial Biology, J. Cardiovasc. Pharmacol., Vol.21 (1993), pp. S6-S10.

(7) Sprague, E.A., Steinbach, B.L., Nerem, R.M. and Schwartz, C.J., Influence of a Laminar Steady-State Fluid-Imposed Wall Shear Stress on the Binding, Internalization, and Degradation of Low-Density Lipoproteins by Cultured Arterial Endothelium, Circulation, Vol.76 (1987), pp.648-656.

( 8 ) Davies, P.F., Dewey, C.F., Jr., Bussolari, S.R., Gordon, E.J. and Gimbrone, M.A., Jr., Influence of Hemodynamic Forces on Vascular Endothelial Function. In 
Vitro Studies of Shear Stress and Pinocytosis in Bovine Aortic Cells, J. Clin. Invest., Vol.73 (1984), pp.11211129.

( 9 ) Jo, H., Dull, R.O., Hollis, T.M. and Tarbell, J.M., Endothelial Albumin Permeability Is Shear Dependent, Time Dependent, and Reversible, Am. J. Physiol., Vol.260 (1991), pp.H1992-H1996.

(10) Ohshima, N. and Ookawa, K., Effect of Shear Stress on Cytoskeletal Structure and Physiological Functions of Cultured Endothelial Cells, Cell Mechanics and Cellular Engineering., Edited by Mow, V.C., Guilak, F., Tran-Son-Tay, R. and Hochmuth, R.M., (1994), pp.107-117, Springer-Verlag, New York.

(11) Kudo, S., Ikezawa, K., Matsumura, S., Ikeda, M., Oka, K. and Tanishita, K., Effect of Shear Stress on Macromolecule Uptake into Cultured Endothelial Cells, Trans. Jpn. Soc. Mech. Eng., (in Japanese), Vol.97, No.892, B (1998), pp.367-374.

(12) Schnitzer, J.E., Update on the Cellular and Molecular Basis of Capillary Permeability, Trends. Cardiovasc. Med., Vol.3 (1993), pp.124-130.

(13) Simionescu, M. and Simionescu, N., Endothelial Transport of Macromolecules: Transcytosis and Endocytosis. A look from Cell Biology, Cell Biol. Rev., Vol.25 (1991), pp.1-78.

(14) Ghitescu, L. and Bendayan, M., Transendothelial Transport of Serum Albumin: A Quantitative Immunocytochemical Study, J. Cell Biol., Vol.117 (1992), pp.745-755.

(15) Schnitzer, J.E. and Oh, P., Albondin-Mediated Capillary Permeability to Albumin, J. Biol. Chem., Vol.269 (1994), pp.6072-6082.

(16) Schnitzer, J.E., Oh, P., Pinny, E. and Allard, J., FilipinSensitive Caveolae-Mediated Transport in Endothelium - Reduced Transcytosis, Scavenger Endocytosis, and Capillary Permeability of Select Macromolecules —, J. Cell Biol., Vol.127 (1994), pp.1217-1232.

(17) Shasby, D.M. and Shasby, S.S., Active Transendothelial Transport of Albumin - Interstitium to Lumen Circ. Res., Vol.57 (1985), pp.903-908.

(18) Siflinger-Birnboim, A., Del Vecchio, P.J., Cooper, J.A. and Malik, A.B., Transendothelial Albumin Flux: Evidence against Asymmetric Transport, J. Appl. Physiol., Vol.61 (1986), pp.2035-3039.

(19) Gruenberg, J., Griffiths, G. and Howell, K.E., Characterization of the Early Endosome and Putative Endocytic Carrier Vesicles in Vivo and with an Assay of Vesicle Fusion in Vitro, J. Cell Biol., Vol.108 (1989), pp.1301-1316.

(20) Kelly, R.B., Microtubules, Membrane Traffic, and Cell Organization, Cell, Vol.61 (1990), pp.5-7.

(21) Terasaki, M., Chen, L.B. and Fujiwara, K., Microtubules and the Endoplasmic Reticulum Are Highly Interdependent Structures, J. Cell Biol., Vol.103 (1986), pp.1557-1568.

(22) Liu, S.M., Magnusson, K.E. and Sundqvist, T., Microtubules Are Involved in Transport of Macromolecules by Vesicles in Cultured Bovine Aortic Endothelial Cells, J. Cell Physiol., Vol.156 (1993), pp.311-316.

(23) Antohe, F., Dobrila, L., Heltianu, C., Simionescu, N. and Simionescu, M., Albumin-Binding Proteins Function in the Receptor-Mediated Binding and Transcytosis of Albumin across Cultured Endothelial Cells, Eur. J. Cell Biol., Vol.60 (1993), pp.268-275.

(24) Levesque, M.J. and Nerem, R.M., The Elongation and Orientation of Cultured Endothelial Cells in Response to Shear Stress, J. Biomech. Eng., Vol.107 (1985), pp.341-347.

(25) Ichimura, T., Visualization of Endosomes and Their Evolution, Seitai No Kagaku, (in Japanese), Vol.47 (1996), pp.268-275.

(26) Mundy, D.I., Machleid, T., Ying, Y.-S., Anderson, R.G.W. and Bloom, G.S., Dual Control of Caveolar Membrane Traffic by Microtubules and the Actin Cytoskelton, J. Cell Sci., Vol.115 (2002), pp.4327-4339.

(27) Murray, J.W., Bananis, E. and Wolkoff, A.W., Reconstitution of ATP-Dependent Movement of Endocytic Vesicles along Microtubules in Vitro: An Oscillatory Bidirectional Process, Mol. Biol. Cell, Vol.11 (2000), pp.419-433.

(28) Bananis, E., Murray, J.W., Stockert, R.J., Satir, P. and Wolkoff, A.W., Regulation of Early Endocytic Vesicle Motility and Fission in a Reconstitued System, J. Cell Sci., Vol.116 (2003), pp.2749-2761.

(29) Kudo, K., Ikezawa, K., Matsumura, S., Ikeda, M., Oka, K. and Tanishita, K., Relationship between Energy-Dependent Macromolecule Uptake and Transport Granules in the Endothelial Cells Affected by Wall Shear Stress, JSME Int. J., Ser. C, Vol.42, No.3 (1999), pp.706-714.

(30) Kudo, S., Morigaki, R., Saito, J., Ikeda, M., Oka, K. and Tanishita, K., Shear-Stress Effect on Mitochondrial Membrane Potential and Albumin Uptake in Cultured Endothelial Cells, Biochem. Biophys. Res. Commun., Vol.270 (2000), pp.616-621. 